\title{
Bacterial Analysis by MALDI-TOF Mass Spectrometry: An Inter-Laboratory Comparison
}

\author{
Sharon C. Wunschel, Kristin H. Jarman, Catherine E. Petersen, \\ Nancy B. Valentine, and Karen L. Wahl \\ Pacific Northwest National Laboratory, Richland, Washington, USA
}

Dunja Schauki and Joany Jackman

Johns Hopkins University Applied Physics Laboratory, Laurel, Maryland, USA

\author{
Chad P. Nelson* and Edward White V \\ National Institute of Standards and Technology, Gaithersburg, Maryland, USA
}

\begin{abstract}
Bacterial analysis by matrix-assisted laser desorption/ionization time-of-flight (MALDI-TOF) mass spectrometry has been demonstrated in numerous laboratories, and a few attempts have been made to compare results from different laboratories on the same organism. It has been difficult to understand the causes behind the observed differences between laboratories when different instruments, matrices, solvents, etc. are used. In order to establish this technique as a useful tool for bacterial identification, additional efforts in standardizing the methods by which MALDI mass spectra are obtained and comparisons of spectra from different instruments with different operators are needed. Presented here is an extension of our previous single-laboratory reproducibility study with three different laboratories in a controlled experiment with aliquots of the same bacterial culture, matrix stock solution, and calibrant standards. Using automated spectral collection of whole-cell bacteria and automated data processing and analysis algorithms, fingerprints from three different laboratories were constructed and compared. Nine of the ions appeared reproducibly within all three laboratories, with additional unique ions observed within each of the laboratories. An initial evaluation of the ability to use a fingerprint generated within one laboratory for bacterial identification of a sample from another laboratory is presented, and strategies for improving identification rates between laboratories is discussed. (J Am Soc Mass Spectrom 2005, 16, 456-462) (c) 2005 American Society for Mass Spectrometry
\end{abstract}

$\mathrm{M}$ atrix-assisted laser desorption/ionization timeof-flight mass spectrometry (MALDI-TOF MS) has been used to analyze intact, cultured microorganisms with minimal sample handling. Two recent review articles, which include the capabilities and current limitations that need to be addressed, provide an excellent overview of this emerging research field [1, 2]. The MALDI-TOF MS technique for identifying biomolecules provides rapid analysis time $(<1 \mathrm{~min}$ per sample analy-

Published online January 29, 2005

Address reprint requests to Dr. K. L. Wahl, Pacific Northwest Laboratory, P.O. Box 999, MS P8-08, Richland, WA 99352, USA. E-mail: Karen.Wahl@ pnl.gov

Disclaimer: Certain commercial equipment, instruments, or materials are identified in this paper to specify adequately the experimental procedure. Such identification does not imply recommendation or endorsement by the National Institute of Standards and Technology, nor does it imply that the materials or equipment identified are the best available for the purpose.

* Currently at the U.S. Food and Drug Administration, College Park, Maryland, USA sis), low sample-volume requirements ( $<1 \mu \mathrm{L}$ fluid), and the highly selective nature of mass-spectrometric analysis based on relative molecular masses. The $\mathrm{m} / \mathrm{z}$ values for mass spectral peaks and the patterns with which they are observed provide very specific and unbiased analysis, as they indicate molecular weights of true components of the sample. Bacterial cells have been identified by comparing MALDI-TOF spectra obtained from cultured bacterial cells and simple microbial mixtures against a library of known MALDI-TOF spectral fingerprints obtained from intact bacterial cells $[3,4]$ or from comparison with masses predicted from a proteomic database $[5,6]$. The proteomic approach has been demonstrated to correctly identify bacteria from spectra originating at different laboratories [5], however, this approach is currently suffering from an incomplete protein database for many of the organisms that are of interest. As the proteomic database becomes more populated with organisms of concern, this approach will become more feasible for bacterial identification, at 
least for relatively pure samples. For these reasons, the research presented here concentrates on the use of the MALDI-TOF MS library approach.

In order to establish this technique as a useful tool for bacterial identification, additional efforts in standardizing the methods by which MALDI mass spectra are obtained and comparisons of spectra from different instruments with different operators are needed. A comparison of spectra in the literature collected in different laboratories from samples of Escherichia coli revealed a number of discrepancies in the ions identified [5, 7-9]. Differences in sample preparation and matrix selection have confounded the ability to make direct comparisons among laboratories. A recent article by Williams et al. [10] discusses the experimental factors that affect the quality and reproducibility of bacterial analysis by MALDI-TOF MS. They incorporate the use of a different laser for ionization which introduces another experimental variable. Previous studies have also lacked sufficient replication to compare the variability in ion presence across replicates both within and between laboratories. There has been a lot of research on the development of standardized sample preparation protocols. The focus of this research is to standardize the data collection and MALDI-MS data analysis methods. This will result in the identification of the similarities and differences among replicate spectra from different laboratories when collected under identical conditions with the same sample preparation. E. coli was selected as the initial model organism for this interlaboratory comparison because it is a well-characterized organism that has been used extensively in MALDI-MS of bacteria development.

Presented here is an extension of our previous singlelaboratory reproducibility study with three different laboratories and three different commercial MALDI-TOF MS instruments in a controlled experiment with aliquots of the same bacterial culture, matrix stock solution, and calibration standards. A single culture of E. coli ATCC 33694 was grown at Johns Hopkins University Applied Physics Laboratory (JHU-APL), aliquoted into replicate sample vials, and distributed to JHU-APL, National Institute of Standards and Technology (NIST), and Pacific Northwest National Laboratory (PNNL) to control for possible differences in growth conditions. A single batch of mass calibrants was prepared at NIST and distributed to the three laboratories to control for possible differences in batches. Also, a single batch of matrix solution was prepared at JHU-APL and distributed for use at all three laboratories. Replicate spectra were collected across days and vials to account for many of the remaining sources of variability.

For most applications and for confidence in statistically-based results, bacterial identification using MALDI-TOF MS requires automated data collection and analysis. Where possible, an attempt was made to automate laborious steps in the process. Once the MALDI sample spots were prepared by the operators, each instrument collected data from fifty predeter- mined or random positions within the spot in an automated fashion. Automated spectral acquisition also reduces the effects of operator bias that can often be encountered when manually searching for sweet spots. The spectra were then sent to PNNL for data analysis. Spectral peaks were identified using a patented automated peak detection algorithm [11] developed at PNNL. Data between laboratories were then compared in the following manner. Sixty replicate spectra were used to create a fingerprint within each laboratory following the procedure described in [3, 13]. Thirty additional spectra collected within each laboratory were compared to all three laboratory fingerprints using an algorithm for automated bacterial identification [3]étoéestimateétheédetectionérateéoféE. coli ATCC 33694 within and among laboratories.

Particularly in field applications, bacterial identification using MALDI-TOF MS will often require comparison of spectra generated on one instrument (i.e., spectra collected on an unknown sample) to spectra generated on a second instrument (i.e., spectra collected for fingerprint generation). Differences in instrumental quality, performance, mass range and mass resolution, tuning parameters, etc., can have a profound effect on the resulting spectrum for a given sample. The research presented here begins to uncover the magnitude of the spectral differences that can be expected when using three commercial instruments under carefully controlled experimental conditions. Through this analysis of the variability in peak presence and location among instruments, and the corresponding influence of this variability on bacterial identification among instruments, we can begin identifying the challenges of the current fingerprinting approach and propose modifications to the fingerprinting protocol and associated algorithms for identification across instrument platforms.

\section{Experimental}

\section{Supplies}

3,5-Dimethoxy-4-hydroxycinnamic acid (sinapinic acid), trifluoroacetic acid (TFA), and high performance liquid chromatography (HPLC) grade water were purchased from Aldrich (Milwaukee, WI). Cytochrome $c$ from bovine heart and angiotensin I were purchased from Sigma (St. Louis, MO). Purified water (18.2 M $\Omega$ ) was prepared using a Millipore Milli-Q system (Bedford, MA). Acetonitrile (ACN) was purchased from J. T. Baker (Phillipsburg, NJ). E. coli ATCC 33694 was obtained from the American Type Culture Collection (Manassas, VA). Tryptic soy agar (TSA) with 5\% sheep's blood was purchased from BioMérieux Micro Diagnostic Laboratory (Marcy-l'Etoile, France).

\section{Safety Precautions}

TFA is corrosive and causes severe burns. It is toxic by inhalation, in contact with skin, and if swallowed. 
Suitable protective clothing including lab coat, gloves and eye/face protection should be worn when working with TFA.

\section{Sample Preparation}

E. coli ATCC 33694 was grown aerobically on a TSA plate with $5 \%$ sheep's blood at $37^{\circ} \mathrm{C}$ for $18 \mathrm{~h}$, harvested with calibrated Difco (Sparks, MD) brand $10 \mu \mathrm{L}$ loops and washed three times with high performance liquid chromatography (HPLC) grade water. The cell concentration was determined by the means of a Petroff Hausser Counter (Hausser Scientific Inc., Horsham, PA) that is a commonly used tool for bacterial enumeration in cells $/ \mathrm{mL}$. The cell suspension was then aliquoted into forty-five $0.5 \mathrm{~mL}$ Sarstedt (Newton, NC) brand vials with a pelleted concentration of $10^{9}$ cells $/ \mathrm{mL}$. The vials were stored at $-80{ }^{\circ} \mathrm{C}$ and fifteen vials were distributed to each laboratory. Just prior to analysis at each laboratory, a pellet was thawed and re-suspended in $200 \mu \mathrm{L}$ HPLC grade water for a final concentration of $2.7 \times 10^{10}$ cells $/ \mathrm{mL}$.

\section{MALDI-TOF MS Analysis}

The bacterial suspensions $(1 \mu \mathrm{L})$ were directly spotted onto a MALDI sample plate and allowed to air dry. Sinapinic acid solution $(20 \mathrm{mg} / \mathrm{mL}$ in $70 \%$ ACN: $30 \%$ water and $0.1 \%$ TFA) was prepared in bulk at JHU-APL and distributed to the three laboratories. One $\mu \mathrm{L}$ of the matrix solution was layered onto each bacterial spot and allowed to air dry. Data were collected on three different commercial time-of-flight mass spectrometers. A Kratos (Chestnut Ridge, NY) MALDI IV was used at JHU-APL, an Applied Biosystems (Foster City, CA) Voyager DE-STR at NIST, and an Applied Biosystems (Foster City, CA) Voyager DE-RP at PNNL. All three instruments were operated with a nitrogen laser (337 $\mathrm{nm}$ ) in the linear and positive ion mode. Laser power was not measured, but independently set in each laboratory and kept constant throughout the study.

MALDI-TOF spectra were collected across three days at each of the laboratories. Each day, at each laboratory, three vials were prepared for analysis according to the sample preparation protocol described above. Ten sample spots were prepared for each of the samples from the three vials within a given day. Thus, for each laboratory, a total of ninety spectra were collected over the three-day period. Each spectrum was created by averaging 50 laser shots collected from a set of predetermined or random positions across a spot. There were instrument limitations as to how these 50 shots could be collected. A pattern spiraling outward from the center was used at PNNL, and NIST utilized a random search methodology. Spots on the JHU-APL sample plate were analyzed in a uniform raster across the length of the rectangular spots traditionally used on the Kratos MALDI IV. Masses were externally calibrated using an angiotensin I and cytochrome $c$ stock solution prepared by NIST. These two calibrants were previously chosen as acceptable for internal calibration to bracket the majority of the ions observed for wholecellé bacterialé analysisé [12].é Externalé calibrationé was chosen in this experiment to test performance in this mode to avoid future interference issues that can occur with the use of internal calibrants, especially with unknown samples. The stock solution was aliquoted into vials and lyophilized to stabilize for shipment to the other laboratories. Each day one standard vial was removed from the freezer at each laboratory and reconstituted with $100 \mu \mathrm{L}$ of solvent (30\% ACN:70\% water and $0.01 \%$ TFA) also provided. For each vial, $1 \mu \mathrm{L}$ of standard solution was mixed with $24 \mu \mathrm{L}$ matrix solution to produce a final concentration of $3 \mathrm{pmol} / \mu \mathrm{L}$ of angiotensin I and cytochrome $c, m / z 1297.5$ and 12,231, respectively. External calibration files were created with a fresh calibrant solution every day. At least one calibration spot was used for each group of ten spots within a day. PNNL and NIST sample plates could move in two dimensions allowing the calibration spot to be centrally located near the middle of each group of ten spots for external calibration. One calibration spot was centered between the ten spots on the JHU-APL plate to account for mass drift across the linear sample plate.

\section{Results and Discussion}

The data from all three laboratories were compiled and analyzed at PNNL using their automated peak extraction, fingerprint generation and bacterial identification procedures, for determination of within-laboratory reproducibility and between-laboratory comparisons. Major concerns for this application include the reproducibility of the MALDI signatures for a single bacterial sample and the ability to obtain similar spectra from different laboratories with different instruments. A set ofésampleéspectraéareépresentedéinéFigureél.éVisually,
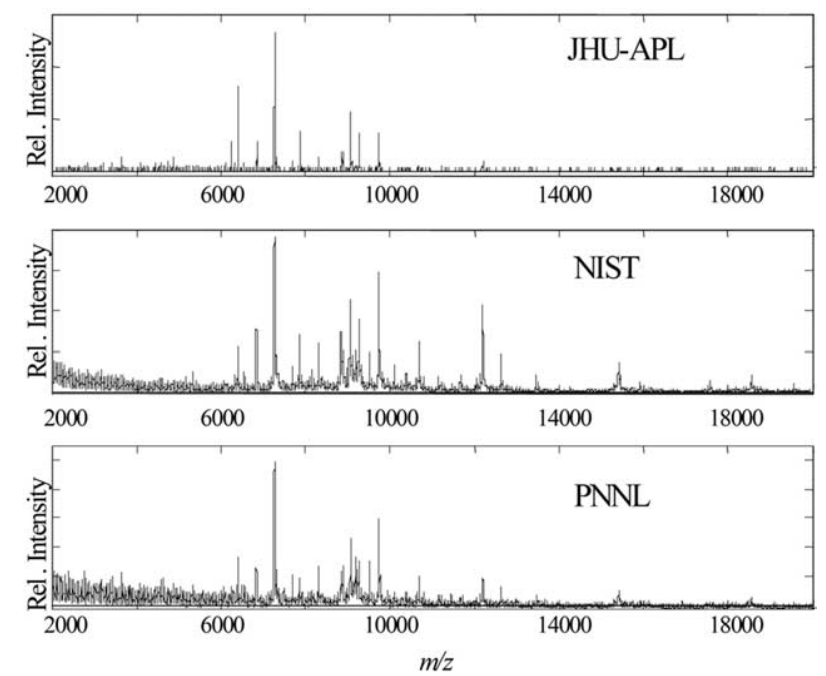

Figure 1. Representative E. coli (ATCC 33694) 50 shot average spectra from the three laboratories. 
the spectra looked quite different from one instrument to another in terms of relative peak intensities and general profile, however when analyzed by the PNNL algorithm, the $m / z$ of many of the ions were similar. The PNNL algorithm for bacterial identification utilizes ion presence/absence and is robust to the variability in ion intensityé [11].é Aé subseté ofé theé 90 é replicateé spectra collected at each laboratory was pulled aside to be used as a test set. Sixty spectra from each laboratory (two out of the three vials for each day) were combined to create aelaboratoryếfingerprintéaséoutlinedênêarmanéetél.A3]. These fingerprints will be referred to as the JHU-APL, NIST, and PNNL fingerprints. The fingerprint for each laboratory consists of the set of reproducible ions observed in at least $70 \%$ of the replicate spectra for that laboratory. The empirically selected $70 \%$ threshold in the PNNL fingerprinting protocol provided the maximum same-species degree of association and minimum across-species degree of association for the fingerprints inétheéPNNLéreferenceélibraryé[13].éTheéfingerprint captures and accounts for the variability in peak $\mathrm{m} / \mathrm{z}$ and peak presence of the ions. Nine fingerprint ions were common across all three laboratory fingerprints Figuresé2éandé3).éFigureé4écontainséaéclose-upéviewéof the $\mathrm{m} / \mathrm{z}$ region surrounding four of the more prominent ions as an example of the similarities and differences between the three laboratories. By narrowing in on this smaller region, the variability in peak location is more apparent both within and between instruments. The region between $\mathrm{m} / \mathrm{z} 6200$ and 8000 was selected as a sample region based on the consistent appearance of four common peaks. This region also shows the appearance of additional peaks reproducibly observed in individual laboratories, but not reproducibly observed across all three laboratories. The $\mathrm{m} / \mathrm{z}$ of all peaks between $\mathrm{m} / \mathrm{z} 6200$ and 8000 from the ninety replicates within each laboratory are plotted. Four of the peaks in this region are common to all three laboratory fingerprints and are highlighted with a corresponding oval. The table to the right of the plot contains peak summary
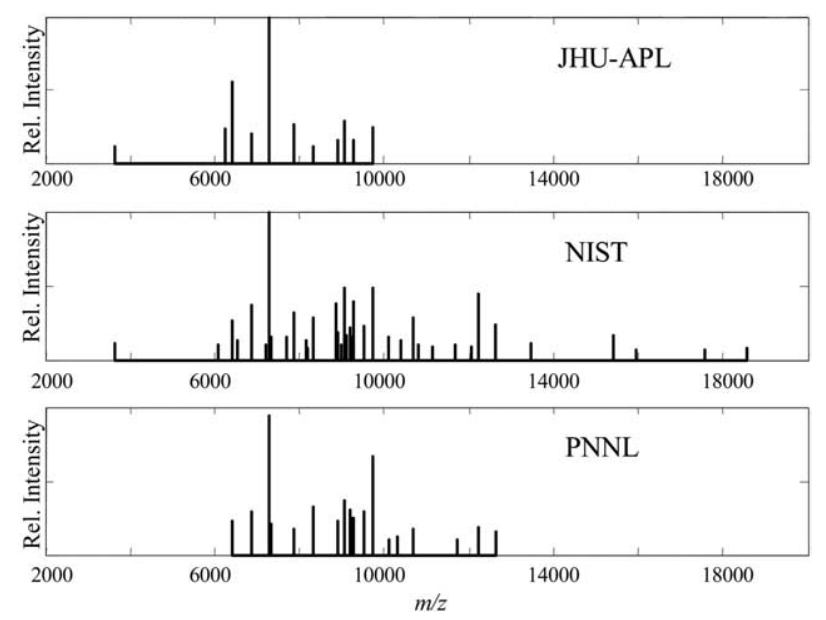

Figure 2. The laboratory fingerprints of E. coli (ATCC 33694).

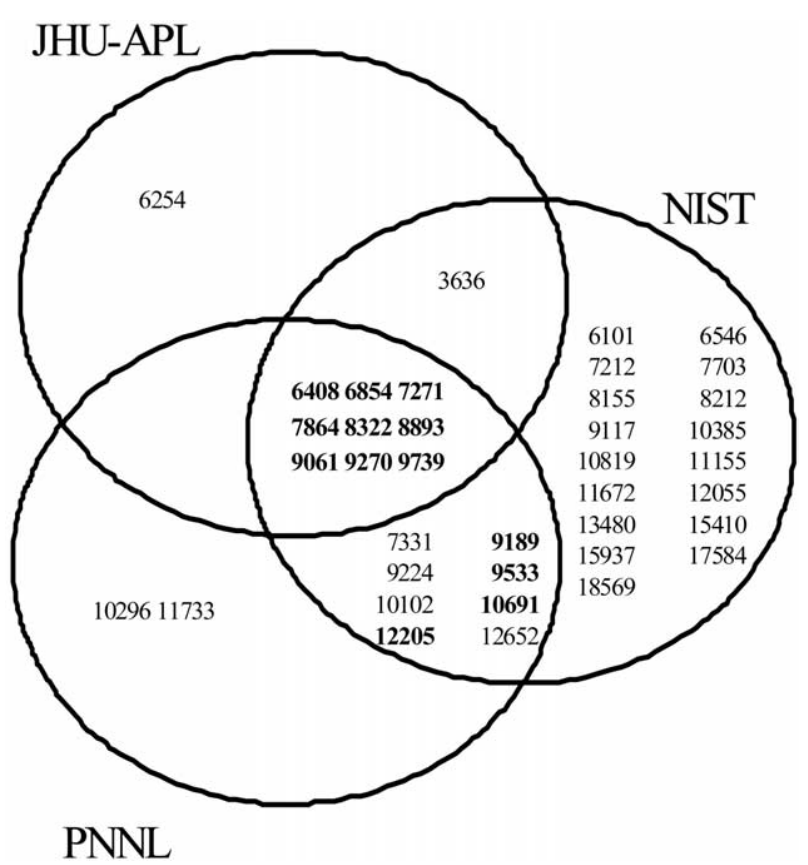

Figure 3. A Venn diagram illustrating the overlap of ions in the lab-level fingerprints, constructed from 60 replicate spectra within each laboratory. Ions in bold represent the set of ions selected for the tri-laboratory fingerprint.

statistics for the four common ions. Specifically, the table contains the mean $\mathrm{m} / \mathrm{z}$ of the replicates identified by the PNNL peak detection algorithm for each laboratory, the corresponding standard deviation, and the percent of the spectra in which the peak was identified as present. This illustrates the strong consistency of certain ions independent of laboratory, despite the apparent differences between the observed spectra, and demonstrates the strength of the algorithm in extracting peaks.

In spite of the fingerprint commonalities, there were a number of ions appearing in only one or two of the fingerprints. This difference is not altogether surprising, since three different instruments were used in the study. The upper-level research-grade MALDI-TOF MS instrument used at NIST found the largest number of ions reproducibly from this sample across the mass range. Fewer high-mass ions were observed reproduciblyéwithétheéinstrumentéatéJHU-APLé(Figureé2).éThis could be in part due to instrument performance and design and in part to current individual tuning and calibration. As expected, there was also some irregularity in the standard deviation of the $\mathrm{m} / \mathrm{z}$ estimates betweenétheélaboratoriesé(Figureé4).éThisédifferenceéin $\mathrm{m} / \mathrm{z}$ variability poses a significant challenge for agent identification between instruments. The estimated standard deviations in the fingerprint are used to predict the $\mathrm{m} / \mathrm{z}$ window around each peak for identification of common peaks in future samples. If the variability in the $m / z$ on the instrument exceeds the variability in the fingerprint estimate, the presence of the fingerprint peaks in a future sample will often be missed. None- 


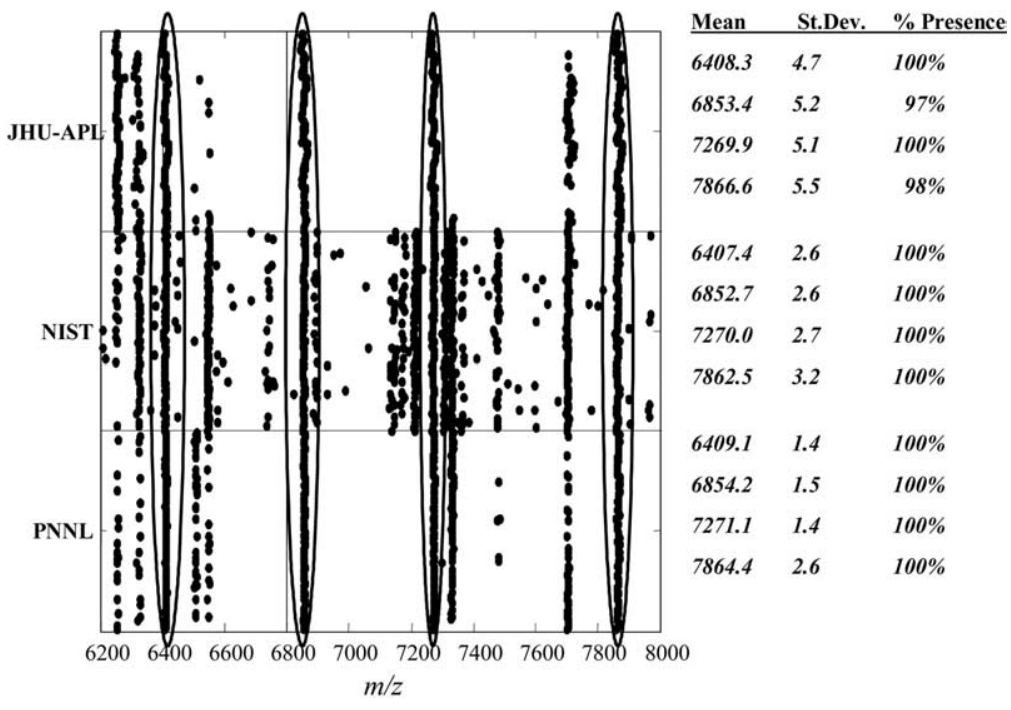

Figure 4. A plot illustrating the variability in peak $\mathrm{m} / \mathrm{z}$ of peaks $(\mathrm{m} / \mathrm{z} 6200$ to 8000$)$ within and between laboratories. The ovals encase the plotted $\mathrm{m} / \mathrm{z}$ of four peaks common to all three laboratory fingerprints. The table to the right provides summary statistics of the estimated mean, standard deviation and percent presence within each laboratory.

theless, inter-laboratory bacterial identification was achieved even when the fingerprint and test spectra originatedéatédifferentelaboratoriesé(Tableél).êTheéidentificationératesênêTableélévereécreatedêbyécomparingểhe remaining 30 spectra collected from each laboratory to all three fingerprints using the methodology outlined in Jarmanéetéal.é[3].êTheéalgorithmsécorrectlyéidentifiedéE. coli in close to $100 \%$ of the test samples within each laboratory. One spectrum from NIST failed to identify E. coli because of an $\mathrm{m} / \mathrm{z}$ shift that exceeded the expected variability in $\mathrm{m} / \mathrm{z}$ as estimated in the NIST fingerprint. Identification of E. coli in the test samples was generally achieved more frequently when the test samples were compared to the JHU-APL fingerprint. This fingerprint contained the highest percentage of overlapping ions $(82 \%)$, even though it had the fewest ions. The test spectra from JHU-APL were challenged by the presence of the additional ions and tighter estimates of the variability in $m / z$ in the PNNL and NIST fingerprints. Seven of the spectra collected at NIST contained a sufficient representation of PNNL fingerprint ions for identification, but the $\mathrm{m} / \mathrm{z}$ of the corresponding peaks were shifted outside of the anticipated window and the identification was not made.

In addition to providing insight on the spectral differences observed among laboratories, this study has provided an evaluation of our current algorithms for within-laboratory comparisons. We can now begin to identify the limitations of these algorithms and initiate modifications for between-laboratory comparisons and the development of a more robust fingerprint library. Combined with the observed differences in the quantity and uniqueness of fingerprint ions, additional challenges were encountered in recognizing common peaks between laboratories because of additional variability in the $m / z$ estimates observed. A number of approaches for improving the identification rate when multiple instruments are used immediately come to mind.

One possible approach involves modifying the algorithms to incorporate the instrument differences into the comparison. For example, the mass resolution and mass accuracy of the instruments could be included as additional parameters to assist in aligning the peaks in an unknown sample with the fingerprint peaks of a similar $\mathrm{m} / \mathrm{z}$. One of the biggest challenges faced with the fingerprinting approach in general is carefully controlled mass axis calibration. Additional calibrant ions may help reduce some of the variability in the $\mathrm{m} / \mathrm{z}$ values observed. The two-component calibration mix was initially chosen as an internal calibration for earlier PNNL library development to minimize interference from the samples. Another issue observed with the data presented here is the difference in mass range of ions observed from the three different instruments. To account for differences in the optimal mass range between instruments, fingerprint peaks outside of the optimal mass windows for the instrument collecting the unknown samples could be down-weighted. One advantage of this approach is that the fingerprints could be generated on a single instrument and compared to unknown samples collected on separate instruments. The disadvantage is that it is hard to identify and account for all of the differences between instruments.

To mediate differences in the number of ions observed between instruments, an alternative approach would be to define the fingerprint to include the minimum number of possible ions observed from any one instrument. For example, one might select the fingerprint with the fewest number of ions to represent the organism. This would ensure that a significant number of fingerprint ions could be expected from an unknown sample regardless of the instrument. However, this 
Table 1. The percent identification when test data within a laboratory were compared to each of the three laboratory fingerprints

\begin{tabular}{|c|c|c|c|c|c|}
\hline \multirow{2}{*}{\multicolumn{2}{|c|}{ Percent Identification (30 spectra) }} & \multicolumn{3}{|c|}{ Fingerprint origination } & \multirow[b]{2}{*}{ Tri-lab } \\
\hline & & JHU-APL & NIST & PNNL & \\
\hline \multirow{3}{*}{$\begin{array}{l}\text { Test data } \\
\text { origination }\end{array}$} & JHU-APL & 100 & 70 & 70 & 100 \\
\hline & NIST & 100 & 97 & 77 & 100 \\
\hline & PNNL & 100 & 100 & 100 & 100 \\
\hline
\end{tabular}

The within laboratory identification rates appear in bold.

approach might result in an increased number of false positives with instruments yielding a greater number of ions, particularly among bacterial samples that are quite similar such as strains of the same organism. Alternatively, one might choose to bracket the set of anticipated ions for an organism using the instrument generating the fewest number of ions and the instrument generating the greatest number of ions. The advantage of this approach is that, once selected, only two instruments would be required for fingerprint generation. These approaches are presented as areas of future research and would require a more elaborate study characterizing the number of ions in fingerprints for additional instruments, with additional organisms, and with less control of the samples.

The approach we have investigated involves combining spectra from multiple laboratories to form an inter-laboratory fingerprint. Although this approach would require heavier involvement from multiple laboratories, it has the advantage of capturing the additional variability in $m / z$ and frequency of appearance of fingerprint ions encountered among laboratories. If a representative sample of laboratories and instruments are included in the library generation, it should be relatively straightforward for any laboratory to use the library for comparisons.

As a test of this inter-laboratory fingerprinting approach, the 60 spectra from each laboratory used in fingerprint development were combined to form a tri-laboratory fingerprint. A relatively simplistic extension of our current fingerprinting methodology was implemented for library generation. Peaks from the 180 spectra collected for fingerprinting from the three laboratories satisfying the fingerprint reproducibility criterion $(>70 \%$ presence rate) were included in the trilaboratory fingerprint. This included the set of nine fingerprint peaks common across all three laboratory fingerprints and four additional peaks $(\mathrm{m} / \mathrm{z}$ 9189, 9533, 10691, and 12205) shared by the NIST and PNNL fingerprints, and appearing fairly reproducibly (but less than $70 \%$ ) within the JHU-APL samples. The test spectra used in the previous analysis of the individual JHU-APL, NIST, and PNNL fingerprints, 30 samples from each laboratory, were then compared to this tri-laboratory fingerprint. E. coli was correctly identified in $100 \%$ of the test spectra from all three laboratories.

The inter-laboratory fingerprint results presented here are very encouraging and demonstrate the poten- tial of this approach. Despite the differences in the frequency of ion appearance and the variability in $\mathrm{m} / \mathrm{z}$ between instruments, test samples generated on all three instruments correctly identified the sample organism. The data and results generated through this study will be used to further investigate improvements to inter-laboratory fingerprint generation and identification. The additional variability encountered by the inclusion of data from multiple instruments poses even greater challenges in differentiating between bacterial strains. Thus, special attention will need to be paid to the specificity of fingerprinting approaches and identification algorithms that attempt to incorporate these instrumental differences. The results presented were generated from a very controlled experiment using a single organism. Additional research will address expanding this approach with experiments involving multiple organisms, including simple mixtures. Furthermore, the influence of less-controlled sample preparation methods on spectral differences between laboratories will be investigated. A recent study on the effect of culture conditions on the MALDI-TOF mass spectrum of bacterial cells within one laboratory demonstrated the ability to still perform identification under lessécontrolledécultureéconditionsé[14].éAéfutureéstep will be to test data from an independent instrument not used in fingerprint generation with samples not cultured under identical conditions.

\section{Conclusions}

Comparison of MALDI-TOF spectra of the same organism between laboratories has previously been confounded by different sample preparation conditions such as matrix, solvents and wash conditions. Direct comparison between laboratories may require the use of a standard protocol. By using common preparations of E. coli ATCC 33694, calibrants, and matrix, we were able to begin isolating the effects of laboratory and instrument variability. Furthermore, automated spectral acquisition and data analysis were used to satisfy some of the constraints imposed in application. Despite different laboratory conditions and instruments, a collection of common ions was observed reproducibly across all three laboratories when the standard protocol was followed. Additional ions, unique to a laboratory, were also identified, particularly with the higher-end research-grade instrument. Within a given laboratory, 
correct bacterial identification was achieved for 90 to $100 \%$ of the test spectra. Between laboratories, a higher degree of identification was generally achieved when comparing the test data to the JHU-APL fingerprint containing the highest percentage of overlapping ions $(82 \%)$. The comparison of the test spectra to a trilaboratory fingerprint, comprised of data from all three laboratories, yielded even more favorable rates of positive identification at $100 \%$ for each of the three laboratories. This study provides further evidence to support the feasibility of MALDI-TOF MS as a rapid technique for bacterial identification and the potential for using a bacterial library with different instruments.

\section{Acknowledgments}

The research was funded in part by the Defense Advanced Research Projects Agency. Pacific Northwest National Laboratory is operated for the U.S. Department of Energy by Battelle Memorial Institute under contract DE-AC06-76-RLO 1830. The research was also funded in part by the National Institute of Standards and Technology and the Defense Threat Reduction Agency, IACRO 02-4025.

\section{References}

1. Fenselau, C.; Demirev, P. A. Characterization of Intact Microorganisms by MALDI Mass Spectrometry. Mass Spectrom. Rev. 2001, 20, 157-171.

2. Lay, J. O. J. MALDI-TOF Mass Spectrometry of Bacteria. Mass Spectrom. Rev. 2001, 20, 172-194.

3. Jarman, K. H.; Cebula, S. T.; Saenz, A. J.; Petersen, C. E.; Valentine, N. B.; Kingsley, M. T.; Wahl, K. L. An Algorithm for Automated Bacterial Identification Using Matrix-Assisted Laser Ddesorption/Ionization Mass Spectrometry. Anal. Chem. 2000, 72, 1217-1223.

4. Wahl, K. L.; Wunschel, S. C.; Jarman, K. H.; Valentine, N. B.; Petersen, C. E.; Kingsley, M. T.; Zartolas, K. A.; Saenz, A. J. Analysis of Microbial Mixtures by Matrix-Assisted Laser Desorption/Ionization Time-of-Flight Mass Spectrometry. Anal. Chem. 2002, 74, 6191-6199.
5. Demirev, P. A.; Ho, Y.; Ryzhov, V.; Fenselau, C. Microorganism Identification by Mass Spectrometry and Protein Database Searches. Anal. Chem. 1999, 71, 2732-2738.

6. Pineda, F. J.; Lin, J. S.; Fenselau, C.; Demirev, P. A. Testing the Significance of Microorganism Identification by Mass Spectrometry and Proteome Database Search. Anal. Chem. 2000, 72, 3739-3744.

7. Wang, Z. P.; Russon, L.; Li, L.; Roser, D. C.; Long, S. R. Investigation of Spectral Reproducibility in Direct Analysis of Bacteria Proteins by Matrix-Assisted Laser Desorption/Ionization Time-of-Flight Mass Spectrometry. Rapid Commun. Mass Spectrom. 1998, 12, 456-464.

8. Arnold, R.; Reilly, J. Fingerprint Matching of E. coli Strains with Matrix-Assisted Laser Desorption/Ionization Time-of-Flight Mass Spectrometry of Whole Cells Using a Modified Correlation Approach. Rapid Commun. Mass Spectrom. 1998, 12, 630-636.

9. Dai, Y. Q.; Li, L.; Roser, D. C.; Long S. R. Detection and Identification of Low-Mass Peptides and Proteins from Solvent Suspensions of Escherichia coli by High Performance Liquid Chromatography Fractionation and Matrix-Assisted Laser Desorption/Ionization Mass Spectrometry. Rapid Commun. Mass Spectrom. 1999, 13, 73-78.

10. Williams, T. L.; Andrzejewski, D.; Lay, J. O., Jr.; Musser, S. M. Experimental Factors Affecting the Quality and Reproducibility of MALDI TOF Mass Spectra Obtained from Whole Bacteria Cells. J. Am. Soc. Mass Spectrom. 2003, 14, 342-351.

11. Jarman, K. H.; Daly, D. S.; Anderson, K. K.; Wahl, K. L. A New Approach to Automated Peak Detection. Chemom. Intell. Lab. Sys. 2003, 69, 61-76.

12. Saenz, A. J.; Petersen, C. E.; Valentine, N. B.; Gantt, S. L.; Jarman, K. H.; Kingsley, M.; Wahl, K. L. Reproducibility of Matrix-Assisted Laser Desorption/Ionization Time-of-Flight Mass Spectrometry for Replicate Bacterial Culture Analysis. Rapid Commun. Mass Spectrom. 1999, 13, 1580-1585.

13. Jarman, K. H.; Daly, D. S.; Petersen, C. E.; Saenz, A. J.; Valentine, N. B.; Wahl, K. L. Extracting and Visualizing Matrix-Assisted Laser Desorption/Ionization Time-of-Flight Mass Spectral Fingerprints. Rapid Commun. Mass Spectrom. 1999, 13, 1586-1594.

14. Valentine, N. B., Wunschel, S. C., Wunschel, D. S., Petersen, C. E., Wahl, K. L. The Effect of Culture Conditions on Microorganism Identification by MALDI Mass Spectrometry. Applied Environ. Microbiol. 2005, in press. 